\title{
Inhalt
}

Der operative Befehl № 00447: Sein Schicksal in der Provinz ……......................... 9

1. Die Durchführung des Befehls № 00447. Ein Forschungsprojekt ........................ 12

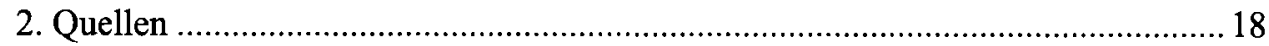

3. Das „Belastungsmaterial“ gegen den NKVD ……............................................ 22

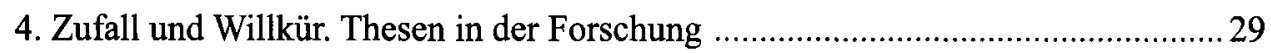

5. Die Durchführung des Befehls № 00447. Eine Gesamtbilanz ……….................. 37

Die Umsetzung des Befehls № 00447: Regionale Perspektiven .................................. 53

OPFER: KuLAKEN, KRIMINELLE UND ANDERE KONTERREVOLUTIONÄRE ELEMENTE .................. 65

Viktor Razgon

Die Verfolgung ehemaliger „Kulaken“ in der Altaj-Region 1937-1938 ...................67

Evgenija Jusopova

Vorgehen gegen die Teilnehmer des Aufstands von Sorokino in der Altaj-

Region

Andrej Suslov

Sondersiedler als Opfer der „Kulakenoperation“ im Rayon Perm’ des Gebiets

Sverdlovsk

Ina Serëgina

Die Untersuchungsakten der Bauernschaft des Gebiets Kalinin als historische

Quelle

Rolf Binner und Marc Junge

Vom „sozial nahen“ zum „sozial feindlichen Element“. Kriminelle in der

sowjetischen Gesellschaft 1918-1938

Viktor Ivanov

Die Kriminellen als Zielgruppe im Gebiet Leningrad

Tat'jana Leont'eva

Kirchen und Religionsgemeinschaften in den Jahren der „bolschewistischen

Perestrojka" im Gebiet Kalinin

Andrej Kolesnikov

Die Verfolgung der Russischen Orthodoxen Kirche im Altaj

Natal'ja Ablažej

Die ROVS-Operation in der Westsibirischen Region 


\section{Viktorija Vološenko}

Die Verfolgung von ehemaligen Bürgerkriegsgegnern im Donbass 309

Jurij Šapoval

Die Behandlung der „ukrainischen Nationalisten“ im Gebiet Kiev

Ol'ga Dovbnja

Die politische „Färbung“ von Verhafteten im Donbass

Andrej Kabackov

Verfolgungen von Arbeitern in der Kama-Region des Gebiets Sverdlovsk

TÄter: StaATs- Und Parteiorgane

Aleksej Tepljakov

Die Rolle des NKVD der Westsibirischen Region

Oleg Lejbovič

Der UNKVD und die „Kulakenoperation“ in der Kama-Region des Gebiets

Sverdlovsk

Aleksandr Čaščuchin

Staatliche Organe und die „Kulakenoperation“

Rolf Binner und Marc Junge

Gutachten der Dorfräte als Routinefaktor bei der Verurteilung von Bauern 503

Irina Gridunova

Rehabilitierungen in der Altaj-Region und dem Gebiet Novosibirsk

1939-1941

Irina Smirnova

Spieglung der „Kulakenoperation“ in Parteidokumenten des Gebiets Doneck

Statistik UND Mikrostudien

Galina Ždanova

Statistische Analyse der Durchführung des Befehls № 00447 im Altaj

Vladimir Nikol'skij

Die „Kulakenoperation“ im ukrainischen Donbass

Sergej Papkov

Die „Kulakenoperation“ im Rayon Krasnozerskoe der Westsibirischen

Region

Sergej Sevyrin

Die Verfolgungen im tatarischen Dorf Kojanovo in der Kama-Region des

Gebiets Sverdlovsk

Elena Vinogradova

Die „,Kulakenoperation“ in den Rayons Firovo und Vyšnij Voloček des

Gebiets Kalinin

AutORENVERZEICHNIS

DANKSAGUNG 


\section{Unserem Kollegen, Freund und Mitautor Rolf Binner}

Im Namen des ganzen Projektteams

Marc Junge und Bernd Bonwetsch 
